\title{
On a new class of summation formulas involving the generalized hypergeometric ${ }_{2} F_{2}$ polynomial
}

\author{
Arjun K Rathie' and Adem Kılıçman²*
}

"Correspondence:
akilicman@putra.upm.edu.my
2Department of Mathematics,
Institute of Mathematical Research,
University Putra Malaysia (UPM),
Serdang, Selangor 43400, Malaysia
Full list of author information is
available at the end of the article

\section{*Correspondence:}

Institute of Mathematical Research University Putra Malaysia (UPM),

Full list of author information is

available at the end of the article

\begin{abstract}
The aim of this research paper is to establish a quite general transformation involving the generalized hypergeometric function. Extensions of Kummer's first transformation, Gauss and Kummer summation, and its contiguous results are then applied to obtain a new class of summation formulas involving the generalized hypergeometric ${ }_{2} F_{2}$ polynomial, which have not previously appeared in the literature. A few well-known results obtained earlier by Kim et al. (Int. J. Math. Math. Sci. 2010:309503, 2010; Integral Transforms Spec. Funct. 23(6):435-444, 2012) and Exton have been obtained as special cases of our main findings.
\end{abstract}

MSC: $33 C 15 ; 33 C 20$

Keywords: generalized hypergeometric series; extension of Gauss and Kummer summation theorems; polynomial

\section{Introduction and results required}

The generalized hypergeometric function with $p$ numeratorial and $q$ denominatorial parameters is defined by $[1-3]$

$$
\begin{aligned}
{ }_{p} F_{q}\left[\begin{array}{c}
\alpha_{1}, \ldots, \alpha_{p} ; z \\
\beta_{1}, \ldots, \beta_{q} ;
\end{array}\right] & ={ }_{p} F_{q}\left[\alpha_{1}, \ldots, \alpha_{p} ; \beta_{1}, \ldots, \beta_{q} ; z\right] \\
& =\sum_{n=0}^{\infty} \frac{\left(\alpha_{1}\right)_{n} \cdots\left(\alpha_{p}\right)_{n}}{\left(\beta_{1}\right)_{n} \cdots\left(\beta_{q}\right)_{n}} \frac{z^{n}}{n !}
\end{aligned}
$$

where $(\alpha)_{n}$ denotes the well-known Pochhammer symbol defined for any $\alpha \in \mathbb{C}$ by

$$
(\alpha)_{n}= \begin{cases}\alpha(\alpha+1)(\alpha+2) \cdots(\alpha+n-1) & (n \in \mathbb{N}=\{1,2,3, \ldots\}), \\ 1 & (n=0)\end{cases}
$$

and $\Gamma$ is the well-known Gamma function defined by

$$
\Gamma(s)=\int_{0}^{\infty} e^{-x} x^{s-1} d x \quad(\Re(s)>0) .
$$

For details as regards convergence etc. of ${ }_{p} F_{q}$, we refer to [2].

\section{Springer}

O2014 Rathie and Klliçman; licensee Springer. This is an Open Access article distributed under the terms of the Creative Commons Attribution License (http://creativecommons.org/licenses/by/2.0), which permits unrestricted use, distribution, and reproduction in any medium, provided the original work is properly cited. 
It is interesting to mention here that whenever a generalized hypergeometric function reduces to gamma functions, the results are very important from the application point of view. Thus well-known classical summation theorems such as those of Gauss, Gauss second, Kummer, and Bailey for the series ${ }_{2} F_{1}$; Watson, Dixon, Whipple and Saalschütz for the series ${ }_{3} F_{2}$ and others play an important role in the theory of hypergeometric, generalized hypergeometric series and other branches of applied mathematics.

Recently a good deal of progress has been made in the direction of generalizations and extensions of the above mentioned classical summation theorems. For this, we refer to the research papers $[4,5]$ and the references therein.

This function has been extensively studied in great detail by many authors such as Exton [6] and Slater [3]. Here we shall mention some of the classical summation theorems, their generalizations, and their extensions so that the paper can be self-contained: Gauss' summation theorem $[2,7]$ :

$$
{ }_{2} F_{1}\left[\begin{array}{cc}
a, b ; & 1 \\
c ; & 1
\end{array}\right]=\frac{\Gamma(c) \Gamma(c-a-b)}{\Gamma(c-a) \Gamma(c-b)},
$$

provided that $\Re(c-a-b)>0$.

Kummer's summation theorem [2, 7]:

$$
{ }_{2} F_{1}\left[\begin{array}{c}
a, b ; \\
1+a-b ;
\end{array}-1\right]=\frac{\Gamma\left(1+\frac{1}{2} a\right) \Gamma(1+a-b)}{\Gamma(1+a) \Gamma\left(1+\frac{1}{2} a-b\right)} .
$$

Contiguous Kummer's summation theorems [7]:

$$
\begin{aligned}
& { }_{2} F_{1}\left[\begin{array}{c}
a, b ; \\
a-b ;
\end{array}-1\right]=\frac{\Gamma\left(\frac{1}{2}\right) \Gamma(a-b)}{2^{a}}\left[\frac{1}{\Gamma\left(\frac{1}{2} a\right) \Gamma\left(\frac{1}{2} a-b+\frac{1}{2}\right)}+\frac{1}{\Gamma\left(\frac{1}{2} a+\frac{1}{2}\right) \Gamma\left(\frac{1}{2} a-b\right)}\right], \\
& { }_{2} F_{1}\left[\begin{array}{c}
a, b ; \\
a-b-1 ;
\end{array}\right]=\frac{\Gamma\left(\frac{1}{2}\right) \Gamma(a-b-1)}{2^{a}} \\
& \times\left[\frac{(a-b-1)}{\Gamma\left(\frac{1}{2} a-b\right) \Gamma\left(\frac{1}{2} a+\frac{1}{2}\right)}+\frac{2}{\Gamma\left(\frac{1}{2} a-b-\frac{1}{2}\right) \Gamma\left(\frac{1}{2} a\right)}\right] .
\end{aligned}
$$

Extension of Gauss' summation theorem [4, 7]:

$$
{ }_{3} F_{2}\left[\begin{array}{c}
a, b, d+1 ; \\
c+1, d ;
\end{array}\right]=\frac{\Gamma(c+1) \Gamma(c-a-b)}{\Gamma(c-a+1) \Gamma(c-b+1)}\left[(c-a-b)+\frac{a b}{d}\right],
$$

provided $\Re(c-a-b)>0$ and $d \neq 0,-1,-2, \ldots$.

Extension of Kummer's summation theorem [4]:

$$
\begin{aligned}
{ }_{3} F_{2}\left[\begin{array}{c}
a, b, d+1 ; \\
2+a-b, d ;
\end{array}\right] & = \\
\quad & \frac{\Gamma\left(\frac{1}{2}\right) \Gamma(2+a-b)}{2^{a}(1-b)}\left[\frac{\left(\frac{1+a-b}{d}-1\right)}{\Gamma\left(\frac{1}{2} a\right) \Gamma\left(\frac{1}{2} a-b+\frac{3}{2}\right)}+\frac{\left(1-\frac{a}{d}\right)}{\Gamma\left(\frac{1}{2} a+\frac{1}{2}\right) \Gamma\left(\frac{1}{2} a-b+1\right)}\right] .
\end{aligned}
$$


Extension of contiguous Kummer's theorem (1.7):

$$
\begin{aligned}
{ }_{3} F_{2}\left[\begin{array}{c}
a, b, d+1 ; \\
a-b, d ;
\end{array}\right] & \\
& =\frac{\Gamma\left(\frac{1}{2}\right) \Gamma(a-b)}{2^{a}}\left[\frac{\left(1-\frac{b}{d}\right)}{\Gamma\left(\frac{1}{2} a\right) \Gamma\left(\frac{1}{2} a-b+\frac{1}{2}\right)}+\frac{1}{\Gamma\left(\frac{1}{2} a+\frac{1}{2}\right) \Gamma\left(\frac{1}{2} a-b\right)}\right],
\end{aligned}
$$

for $d \neq 0,-1,-2, \ldots$

We remark in passing that the result (1.10) is a presumably new result.

Proof In order to derive (1.10) we proceed as follows. Denoting the left-hand side of (1.10) by $S$, expressing ${ }_{3} F_{2}$ as a series, after some simplifications, we have

$$
\begin{aligned}
S & =\sum_{n=0}^{\infty} \frac{(a)_{n}(b)_{n}(-1)^{n}}{(a-b)_{n} n !}\left(1+\frac{n}{d}\right) \\
& ={ }_{2} F_{1}\left[\begin{array}{c}
a, b ;-1 \\
a-b ;
\end{array}\right]+\frac{1}{d} \sum_{n=1}^{\infty} \frac{(a)_{n}(b)_{n}(-1)^{n}}{(a-b)_{n}(n-1) !} .
\end{aligned}
$$

Now replacing $n-1=N$ in the second term and using the result $(a)_{n+1}=a(a+1)_{n}$ and simplifying and finally summing up the second series, we get

$$
S={ }_{2} F_{1}\left[\begin{array}{c}
a, b ; \\
a-b ;
\end{array}\right]-\frac{a b}{d(a-b)}{ }_{2} F_{1}\left[\begin{array}{c}
a+1, b+1 ; \\
1+a-b ;
\end{array}\right] .
$$

Now we observe that the first ${ }_{2} F_{1}$ on the right-hand side of (1.11) can be evaluated with the help of the known result (1.6) while the second ${ }_{2} F_{1}$ on the right-hand side of (1.11) can be evaluated with the help of Kummer's summation theorem (1.5), after some simplification, we easily arrive at the right-hand side of (1.10). This completes the proof of (1.10).

Remark In (1.10), if we set $d=a-b-1$ we recover (1.7).

It is not out of place to mention here that in many branches of pure and applied mathematics, engineering, and mathematical physics, the Laguerre polynomial defined by

$$
L_{n}^{(v)}(x)=\frac{(v+1)_{n}}{n !}{ }_{1} F_{1}\left[\begin{array}{c}
-n ; \\
v+1 ;
\end{array}\right]
$$

which is a terminating form of the confluent hypergeometric function ${ }_{1} F_{1}$, has frequently occurred [8, p.268].

On the other hand, by elementary manipulation of series, very recently, Kim et al. [9] have obtained the following interesting and general transformation involving the generalized hypergeometric function viz.:

$$
\begin{aligned}
& \sum_{n=0}^{\infty} \frac{\left(a_{1}\right)_{n} \cdots\left(a_{p}\right)_{n}}{\left(b_{1}\right)_{n} \cdots\left(b_{q}\right)_{n}} \cdot \frac{(d)_{n}}{n !} x^{n} y^{n}{ }_{1} F_{1}\left[\begin{array}{c}
d+n ; \\
f ;
\end{array}\right] \\
& \quad=\sum_{n=0}^{\infty} \frac{(d)_{n}}{(f)_{n}} \cdot \frac{x^{n}}{n !}{ }^{p+2} F_{q}\left[\begin{array}{c}
-n, 1-f-n, a_{1}, \ldots, a_{b} ; y \\
b_{1}, \ldots, b_{q} ;
\end{array}\right] .
\end{aligned}
$$


Using Kummer's first transformation [2], viz.

$$
{ }_{1} F_{1}\left[\begin{array}{l}
a ; \\
c ;
\end{array}\right]=e^{x}{ }_{1} F_{1}\left[\begin{array}{c}
c-a ; \\
c ;
\end{array}\right]
$$

in (1.13) and employing Kummer's summation theorem (1.5) and Gauss' summation theorem (1.4), they have also obtained the following interesting summation formulas involving the Laguerre polynomial viz.:

$$
e^{-x} \sum_{n=0}^{\infty} \frac{x^{n}}{(v+1)_{n}} L_{n}^{(v)}(x)={ }_{0} F_{1}\left[\begin{array}{c}
-; \\
v+1 ;
\end{array}-x^{2}\right],
$$

where $v \neq-1,-2 \ldots$,

$$
\begin{aligned}
& e^{-x} \sum_{n=0}^{\infty} \frac{x^{n}}{(1-v)_{n}} L_{n}^{(v)}(x) \\
& \left.\quad={ }_{1} F_{2}\left[\begin{array}{cc}
\frac{1}{2} v+\frac{1}{2} ; \\
\frac{1}{2}, 1-\frac{1}{2} v ;
\end{array}\right]+x^{2}\right]+\frac{2 x v}{1-v}{ }_{1} F_{1}\left[\begin{array}{cr}
\frac{1}{2} v+1 ; & -x^{2} \\
\frac{3}{2}, \frac{3}{2}-\frac{1}{2} v ;
\end{array}\right],
\end{aligned}
$$

where $v \neq-1,-2, \ldots$,

$$
e^{-x} \sum_{n=0}^{\infty} \frac{(-x)^{n}}{(\mu)_{n}} L_{n}^{(\nu)}(x)={ }_{2} F_{2}\left[\begin{array}{c}
\frac{1}{2} \mu+\frac{1}{2} v, \frac{1}{2} \mu+\frac{1}{2} v+\frac{1}{2} ;-4 x \\
\mu, \mu+v ;
\end{array}\right] .
$$

Remark 1. In fact, the results (1.13), (1.15), (1.16), and (1.17) are the corrected forms of those obtained earlier by Exton [10].

2. For several new and interesting results closely related to (1.15) and (1.16), see Kim et al. [9].

The following extension of Kummer's first transformation (1.14) established by Paris [11] will be required in our present investigation:

$$
e^{-x}{ }_{2} F_{2}\left[\begin{array}{c}
a, 1+d ; \\
b+1, d ;
\end{array}\right]={ }_{2} F_{2}\left[\begin{array}{cc}
b-a, f+1 ; \\
b+1, f ;
\end{array}\right],
$$

where $f=\frac{d(a-b)}{a-d}$.

The aim of this research paper is to first establish a general transformation formula which generalizes (1.13). Then by employing extensions of Gauss' summation theorem, Kummer's summation theorem, and its contiguous results, we establish three new and interesting summation formulas for the generalized hypergeometric ${ }_{2} F_{2}$ polynomial which generalize the results (1.15), (1.16), and (1.17).

We conclude this section by remarking that the results established in this research paper are simple, interesting, easily established, and (potentially) useful. 


\section{Main transformation formula}

The transformation formula involving the generalized hypergeometric function to be established in this paper is

$$
\begin{aligned}
& \sum_{n=0}^{\infty} \frac{\left(a_{1}\right)_{n} \cdots\left(a_{p}\right)_{n}}{\left(b_{1}\right)_{n} \cdots\left(b_{q}\right)_{n}} \frac{(d)_{n}}{n !} x^{n} y^{n}{ }_{2} F_{2}\left[\begin{array}{c}
d+n, e+1 ; \\
f+1, e ;
\end{array}\right] \\
& \quad=\sum_{n=0}^{\infty} \frac{(d)_{n}(e+1)_{n}}{(f+1)_{n}(e)_{n}} \frac{x^{n}}{n !} \times{ }_{p+3} F_{q+1}\left[\begin{array}{c}
-n, 1-e-n,-f-n, a_{1}, \ldots, a_{p} ; \\
-e-n, b_{1}, \ldots, b_{q} ;
\end{array}\right] .
\end{aligned}
$$

Proof In order to prove (2.1), we proceed as follows. If we denote the left-hand side of (2.1) by $S$ and express ${ }_{2} F_{2}$ as a series, we have

$$
S=\sum_{n=0}^{\infty} \sum_{m=0}^{\infty} \frac{\left(a_{1}\right)_{n} \cdots\left(a_{p}\right)_{n}}{\left(b_{1}\right)_{n} \cdots\left(b_{q}\right)_{n}} \cdot \frac{(d)_{n}(d+n)_{m}(e+1)_{m}}{(f+1)_{m}(e)_{m} n ! m !} x^{n+m} y^{n},
$$

which, upon use of the identity

$$
(d)_{n}(d+n)_{m}=(d)_{m+n}
$$

becomes

$$
S=\sum_{m=0}^{\infty} \sum_{n=0}^{\infty} \frac{\left(a_{1}\right)_{n} \cdots\left(a_{p}\right)_{n}}{\left(b_{1}\right)_{n} \cdots\left(b_{q}\right)_{n}} \cdot \frac{(d)_{n+m}(e+1)_{m}}{(f+1)_{m}(e)_{m} n ! m !} x^{n+m} y^{n}
$$

Now replacing $m$ by $m-n$ and making use of a simple manipulation for the double series $[2$, p.56]

$$
\sum_{n=0}^{\infty} \sum_{k=0}^{\infty} A(k, n)=\sum_{n=0}^{\infty} \sum_{k=0}^{n} A(k, n-k)
$$

we have

$$
S=\sum_{m=0}^{\infty} \sum_{n=0}^{m} \frac{\left(a_{1}\right)_{n} \cdots\left(a_{p}\right)_{n}}{\left(b_{1}\right)_{n} \cdots\left(b_{q}\right)_{n}} \cdot \frac{(d)_{m}(e+1)_{m-n}}{(f+1)_{m-n}(e)_{m-n}} \frac{x^{m} y^{n}}{n !(m-n) !} .
$$

Using the series identities

$$
(a)_{m-n}=\frac{(-1)^{n}(a)_{m}}{(1-a-m)_{n}}
$$

and

$$
(m-n) !=\frac{(-1)^{n} m !}{(-m)_{n}}
$$

in the last summation, after little simplifications, we get

$$
S=\sum_{m=0}^{\infty} \frac{(d)_{m}(e+1)_{m}}{(f+1)_{m}(e)_{n} m} \frac{x^{m}}{m !} \sum_{n=0}^{m} \frac{\left(a_{1}\right)_{n} \cdots\left(a_{p}\right)_{n}(-f-m)_{n}(1-e-m)_{n}(-m)_{n}}{\left(b_{1}\right)_{n} \cdots\left(b_{q}\right)_{n}(-e-m)_{n} n !} y^{n} .
$$


Finally, expressing the inner series as a ${ }_{p+3} F_{q+2}(y)$ hypergeometric function, we then easily arrive at the right-hand side of (2.1). This completes the proof of (2.1).

Remark 1. Our main transformation formula (2.1) is a special case of a general transformation formula given by Slater [3, p.60].

2. In our main transformation formula (2.1), if we take $f=e$, we get at once the result due to Kim et al. [9, p.437, Eq. (2.1)].

\section{New class of summation formulas involving the generalized hypergeometric ${ }_{2} F_{2}$ polynomial}

The summation formulas to be established in this section are given by the following theorems.

Theorem 1 The following formula holds true:

$$
\begin{aligned}
& e^{-x} \sum_{n=0}^{\infty} \frac{x^{n}}{n !}{ }_{2} F_{2}\left[\begin{array}{c}
-n, e+1 ; \\
v+2, e ;
\end{array}\right] \\
& \left.\quad={ }_{2} F_{3}\left[\begin{array}{c}
\frac{1}{2} v+\frac{1}{2}, \frac{1}{2} e+1 ; \\
v+1, \frac{1}{2} e, \frac{1}{2} v+\frac{3}{2} ;
\end{array}-x^{2}\right]+\frac{(e-v-1) x}{e(v+2)}{ }_{1} F_{2}\left[\begin{array}{c}
\frac{1}{2} v+1 ; \\
v+2, \frac{1}{2} v+2 ;
\end{array}\right] . x^{2}\right] .
\end{aligned}
$$

Theorem 2 The following formula holds true:

$$
\begin{aligned}
& e^{-x} \sum_{n=0}^{\infty} \frac{(1+v)_{n}}{(1-v)_{n}} \frac{x^{n}}{n !}{ }_{2} F_{2}\left[\begin{array}{c}
-n, e+1 ; \\
v+2, e ;
\end{array}\right] \\
& =\frac{\Gamma\left(\frac{1}{2}\right) \Gamma(1-v)}{e 2^{-v-1}}\left\{\frac{(e-v-1)}{\Gamma\left(\frac{-1}{2} v\right) \Gamma\left(\frac{1}{2}-\frac{1}{2} v\right)}{ }_{2} F_{3}\left[\begin{array}{c}
\frac{1}{2} v+\frac{1}{2}, \frac{1}{2} v+1 ; \\
\frac{3}{2}, \frac{1}{2} v+\frac{3}{2}, \frac{1}{2}-\frac{1}{2} v ;
\end{array}-x^{2}\right]\right. \\
& \left.-\frac{(e-v)}{\Gamma\left(-\frac{1}{2} v-\frac{1}{2}\right) \Gamma\left(1-\frac{1}{2} v\right)}{ }_{2} F_{3}\left[\begin{array}{c}
\frac{1}{2} v+\frac{1}{2}, \frac{1}{2} e-\frac{1}{2} v+1 ; \\
\frac{3}{2}, \frac{1}{2} e-\frac{1}{2} v, 1-\frac{1}{2} v ;
\end{array}\right]\right\} \\
& -x \frac{(v+1)}{(v+2)} \cdot \frac{\Gamma\left(\frac{1}{2}\right) \Gamma(1-v)}{e 2^{-v-1}}\left\{\frac{(e-v-1)}{\Gamma\left(-\frac{1}{2} v-\frac{1}{2}\right) \Gamma\left(1-\frac{1}{2} v\right)}\right. \\
& \times{ }_{3} F_{4}\left[\begin{array}{c}
1, \frac{1}{2} v+1, \frac{1}{2} v+\frac{3}{2} ; \\
2, \frac{3}{2}, \frac{1}{2} v+2,1-\frac{1}{2} v ;
\end{array}-x^{2}\right] \\
& \left.-\frac{(1+e-v)}{\Gamma\left(-\frac{1}{2} v-1\right) \Gamma\left(\frac{3}{2}-\frac{1}{2} v\right)}{ }_{3} F_{4}\left[\begin{array}{c}
1, \frac{1}{2} v+1, \frac{3}{2}+\frac{1}{2} e-\frac{1}{2} v ; \\
2, \frac{3}{2}, \frac{1}{2}+\frac{1}{2} e-\frac{1}{2} v, \frac{3}{2}-\frac{1}{2} v ;
\end{array}-x^{2}\right]\right\} \text {. }
\end{aligned}
$$

Theorem 3 The following formula holds true:

$$
\begin{aligned}
e^{-x} & \sum_{n=0}^{\infty} \frac{(1+v)_{n}}{(\mu)_{n}} \cdot \frac{(-x)^{n}}{n !}{ }_{2} F_{2}\left[\begin{array}{c}
-n, e+1 ; \\
v+2, e ;
\end{array}\right] \\
\quad= & { }_{5} F_{5}\left[\begin{array}{c}
v+1, \frac{1}{2} \mu+\frac{1}{2} v, \frac{1}{2} \mu+\frac{1}{2} v+\frac{1}{2}, e+\frac{1}{2} \mu-\frac{1}{2} A+\frac{1}{2}, e+\frac{1}{2} \mu+\frac{1}{2} A+\frac{1}{2} ; \\
v+2, \mu, \mu+v+1, e+\frac{1}{2} \mu-\frac{1}{2} A-\frac{1}{2}, e+\frac{1}{2} \mu+\frac{1}{2} A-\frac{1}{2}
\end{array}\right],
\end{aligned}
$$

where $A^{2}=(\mu-1+2 e)^{2}-4 e(\mu+v)$. 
Proof In order to establish Theorem 1, we proceed as follows. If we apply the extension of Kummer's first transformation (1.14) to (2.1), we obtain

$$
\begin{aligned}
e^{x} & \sum_{n=0}^{\infty} \frac{\left(a_{1}\right)_{n} \cdots\left(a_{p}\right)_{n}}{\left(b_{1}\right)_{n} \cdots\left(b_{q}\right)_{n}} \frac{(d)_{n}}{n !} x^{n} y^{n}{ }_{2} F_{2}\left[\begin{array}{c}
f-d-n, e+1 ; \\
f+1, e ;
\end{array}\right] \\
\quad & \sum_{n=0}^{\infty} \frac{(d)_{n}(e+1)_{n}}{(f+1)_{n}(e)_{n}} \frac{x^{n}}{n !}{ }^{n}{ }^{2} F_{q+1}\left[\begin{array}{c}
-n, 1-e-n,-f-n, a_{1}, \ldots, a_{p} ; \\
-e-n, b_{1}, \ldots, b_{p} ;
\end{array}\right] .
\end{aligned}
$$

Putting $d=f$ in (3.4), we get

$$
\begin{aligned}
& e^{x} \sum_{n=0}^{\infty} \frac{\left(a_{1}\right)_{n} \cdots\left(a_{p}\right)_{n}}{\left(b_{1}\right)_{n} \cdots\left(b_{q}\right)_{n}} \frac{(f)_{n}}{n !} x^{n} y^{n}{ }_{2} F_{2}\left[\begin{array}{c}
-n, e+1 ; \\
f+1, e ;
\end{array}-x\right] \\
& =\sum_{n=0}^{\infty} \frac{(f)_{n}(e+1)_{n}}{(f+1)_{n}(e)_{n}} \frac{x^{n}}{n !}{ }^{p+3} F_{q+1}\left[\begin{array}{c}
-n, 1-e-n,-f-n, a_{1}, \ldots, a_{p} ; \\
-e-n, b_{1}, \ldots, b_{p} ;
\end{array}\right] .
\end{aligned}
$$

Now, replacing $x$ by $-x$ and putting $f=v+1$, we have

$$
\begin{aligned}
e^{-x} & \sum_{n=0}^{\infty} \frac{\left(a_{1}\right)_{n} \cdots\left(a_{p}\right)_{n}}{\left(b_{1}\right)_{n} \cdots\left(b_{q}\right)_{n}} \frac{(v+1)_{n}}{n !}(-x)^{n} y^{n}{ }_{2} F_{2}\left[\begin{array}{c}
-n, e+1 ; \\
v+2, e ;
\end{array}\right] \\
\quad & \sum_{n=0}^{\infty} \frac{(v+1)_{n}(e+1)_{n}}{(v+2)_{n}(e)_{n}} \frac{(-x)^{n}}{n !} p+3 F_{q+1}\left[\begin{array}{c}
-n, 1-e-n,-v-1-n, a_{1}, \ldots, a_{p} ; y \\
-e-n, b_{1}, \ldots, b_{q} ;
\end{array}\right] .
\end{aligned}
$$

Now, set $p=0, q=1, b_{1}=v+1$, and $y=-1$ in (3.5), and we get

$$
\begin{aligned}
e^{-x} & \sum_{n=0}^{\infty} \frac{x^{n}}{n !}{ }_{2} F_{2}\left[\begin{array}{c}
-n, e+1 ; \\
v+2, e ;
\end{array}\right] \\
\quad= & \sum_{n=0}^{\infty} \frac{(v+1)_{n}(e+1)_{n}}{(v+2)_{n}(e)_{n}} \frac{(-x)^{n}}{n !}{ }_{3} F_{2}\left[\begin{array}{c}
-n,-v-1-n, 1-e-n ; \\
v+1,-e-n ;
\end{array}\right],
\end{aligned}
$$

which is valid for $|x|<\infty$.

Now, it is readily seen that the ${ }_{3} F_{2}$ on the right-hand side of (3.6) can be evaluated with the help of the contiguous extension of Kummer's summation theorem in (1.10) to yield

$$
\begin{aligned}
e^{-x} & \sum_{n=0}^{\infty} \frac{x^{n}}{n !}{ }_{2} F_{2}\left[\begin{array}{c}
-n, e+1 ; \\
v+2, e ;
\end{array}\right] \\
= & \sum_{n=0}^{\infty} \frac{(v+1)_{n}(e+1)_{n}}{(v+2)_{n}(e)_{n}} \frac{(-x)^{n}}{n !}\left[\frac{\Gamma\left(\frac{1}{2}\right) \Gamma(v+1)}{2^{-n} \Gamma\left(-\frac{1}{2} n\right) \Gamma\left(\frac{1}{2} n+v+\frac{3}{2}\right)}\left(1-\frac{v+1+n}{e+n}\right)\right. \\
& \left.+\frac{\Gamma\left(\frac{1}{2}\right) \Gamma(v+1)}{2^{-n} \Gamma\left(-\frac{1}{2} n+\frac{1}{2}\right) \Gamma\left(\frac{1}{2} n+v+1\right)}\right] .
\end{aligned}
$$


Now, separating the terms appearing on the right-hand side of (3.7) into even and odd powers of $x$ and making use of the following elementary identities:

$$
\begin{aligned}
& \Gamma(a-n)=\frac{(-1)^{n} \Gamma(a)}{(1-n)_{n}}, \quad 2 n !=2^{2 n} n !\left(\frac{1}{2}\right)_{n}, \\
& (2 n+1) !=2^{2 n} n !\left(\frac{3}{2}\right), \quad(a)_{2 n}=2^{2 n}\left(\frac{1}{2} a\right)_{n}\left(\frac{1}{2} a+\frac{1}{2}\right)_{n}, \\
& (a)_{2 n+1}=a 2^{2 n}\left(\frac{1}{2} a+\frac{1}{2}\right)_{n}\left(\frac{1}{2} a+1\right)_{n},
\end{aligned}
$$

and after some straightforward calculation, we have

$$
\begin{aligned}
e^{-x} \sum_{n=0}^{\infty} \frac{x^{n}}{n !}{ }_{2} F_{2}\left[\begin{array}{c}
-n, e+1 ; \\
v+2, e ;
\end{array}\right] & \\
= & \sum_{n=0}^{\infty} \frac{\left(\frac{1}{2} v+\frac{1}{2}\right)_{n}\left(\frac{1}{2} e+1\right)_{n}}{(v+1)_{n}\left(\frac{1}{2} e\right)_{n}\left(\frac{1}{2} v+\frac{3}{2}\right)_{n}} \frac{(-1)^{n} x^{2 n}}{n !} \\
& +\frac{(e-1-v) x}{e(v+2)} \sum_{n=0}^{\infty} \frac{\left(\frac{1}{2} v+1\right)_{n}(-1)^{n}}{(v+2)_{n}\left(\frac{1}{2}+2\right)_{n}} \frac{x^{2 n}}{n !} .
\end{aligned}
$$

Finally, summing up the two series on the right-hand side, we easily arrive at the desired result (2.1). This completes the proof of Theorem 1 .

In order to establish Theorem 2, if we set $p=0, q=1, b_{1}=1-v$ and $y=-1$ in (3.5), we have

$$
\begin{aligned}
e^{-x} & \sum_{n=0}^{\infty} \frac{(v+1)_{n}}{(1-v)_{n}} \frac{x^{n}}{n !}{ }_{2} F_{2}\left[\begin{array}{c}
-n, e+1 ; \\
v+2, e ;
\end{array}\right] \\
\quad & \sum_{n=0}^{\infty} \frac{(v+1)_{n}(e+1)_{n}}{(v+2)_{n}(e)_{n}} \frac{(-x)^{n}}{n !}{ }_{3} F_{2}\left[\begin{array}{c}
-n,-v-1-n, 1-e-n ; \\
1-v, e-n ;
\end{array}\right],
\end{aligned}
$$

which is valid for $|x|<\infty$.

It is now easy to see that the ${ }_{3} F_{2}$ on the right-hand side of (3.10) can be evaluated with the help of the extension of Kummer's summation theorem (1.9); we get

$$
\begin{aligned}
e^{-x} & \sum_{n=0}^{\infty} \frac{(v+1)_{n}}{(1-v)_{n}} \frac{x^{n}}{n !}{ }_{2} F_{2}\left[\begin{array}{c}
-n, e+1 ; \\
v+2, e ;
\end{array}\right] \\
= & \frac{\Gamma\left(\frac{1}{2}\right) \Gamma(1-v)}{2^{-v-1}} \sum_{n=0}^{\infty} \frac{(v+1)_{n}(e+1)_{n}(-x)^{n}}{(v+2)_{n}(e)_{n}(e+n) 2^{-n} \Gamma(n+2)} \\
& \times\left\{\frac{(e-v-1)}{\Gamma\left(-\frac{1}{2} n-\frac{1}{2} v\right) \Gamma\left(\frac{1}{2} n-\frac{1}{2} v+\frac{1}{2}\right)}-\frac{(e-v+n)}{\Gamma\left(-\frac{1}{2} n-\frac{1}{2} v-\frac{1}{2}\right) \Gamma\left(\frac{1}{2} n-\frac{1}{2} v+1\right)}\right\} .
\end{aligned}
$$

Now, separating the terms appearing on the right-hand side of (3.11) into even and odd powers of $x$ and making use of the elementary identities given in (3.8) and after some 
straightforward algebra, we get

$$
\begin{aligned}
e^{-x} \sum_{n=0}^{\infty} \frac{(1+v)_{n}}{(1-v)_{n}} \frac{x^{n}}{n !}{ }_{2} F_{2}\left[\begin{array}{c}
-n, e+1 ; \\
v+2, e ;
\end{array}\right] & \\
= & \frac{\Gamma\left(\frac{1}{2}\right) \Gamma(1-v)}{e 2^{-v-1}}\left\{\frac{(e-v-1)}{\Gamma\left(-\frac{1}{2} v\right) \Gamma\left(\frac{1}{2}-\frac{1}{2} v\right)} \sum_{n=0}^{\infty} \frac{\left(\frac{1}{2} v+\frac{1}{2}\right)_{n}\left(\frac{1}{2} v+1\right)_{n}(-1)^{n} x^{2 n}}{\left(\frac{3}{2}\right)_{n}\left(\frac{1}{2} v+\frac{3}{2}\right)_{n}\left(\frac{1}{2}-\frac{1}{2} v\right)_{n} n !}\right. \\
& \left.-\frac{(e-v)}{\Gamma\left(-\frac{1}{2} v-\frac{1}{2}\right) \Gamma\left(1-\frac{1}{2} v\right)} \sum_{n=0}^{\infty} \frac{\left(\frac{1}{2} v+\frac{1}{2}\right)_{n}\left(\frac{1}{2} e-\frac{1}{2} v+1\right)_{n}(-1)^{n} x^{2 n}}{\left(\frac{3}{2}\right)_{n}\left(\frac{1}{2} e-\frac{1}{2} v\right)_{n}\left(1-\frac{1}{2} v\right)_{n} n !}\right\} \\
& -x \frac{(v+1)}{(v+2)} \frac{\Gamma\left(\frac{1}{2}\right) \Gamma(1-v)}{e 2^{-v-1}}\left\{\frac{(e-v-1)}{\Gamma\left(-\frac{1}{2} v-\frac{1}{2}\right) \Gamma\left(1-\frac{1}{2} v\right)}\right. \\
& \times \sum_{n=0}^{\infty} \frac{(1)_{n}\left(\frac{1}{2} v+1\right)_{n}\left(\frac{1}{2} v+\frac{3}{2}\right)_{n}(-1)^{n} x^{2 n}}{(2)_{n}\left(\frac{3}{2}\right)_{n}\left(\frac{1}{2} v+2\right)_{n}\left(1-\frac{1}{2} v\right)_{n} n !} \\
& \left.-\frac{(1+e-v)}{\Gamma\left(-\frac{1}{2} v+1\right) \Gamma\left(\frac{3}{2}-\frac{1}{2} v\right)} \sum_{n=0}^{\infty} \frac{(1)_{n}\left(\frac{1}{2} v+1\right)_{n}\left(\frac{3}{2}+\frac{1}{2} e-\frac{1}{2} v\right)_{n}(-1)^{n} x^{2 n}}{(2)_{n}\left(\frac{3}{2}\right)_{n}\left(\frac{1}{2}+\frac{1}{2} e-\frac{1}{2} v\right)_{n}\left(\frac{3}{2}-\frac{1}{2} v\right)_{n} n !}\right\} .
\end{aligned}
$$

Finally, summing up the four series on the right-hand side, we arrive at the desired result (3.2). This completes the proof of Theorem 2.

In order to prove Theorem 3, if we set $p=0, q=1, b_{1}=\mu$, and $y=1$ in (3.5), we have

$$
\begin{aligned}
e^{-x} & \sum_{n=0}^{\infty} \frac{(1+v)_{n}}{(\mu)_{n}} \frac{(-x)^{n}}{n !}{ }_{2} F_{2}\left[\begin{array}{c}
-n, e+1 ; \\
v+2, e ;
\end{array}\right] \\
= & \sum_{n=0}^{\infty} \frac{(v+1)_{n}(e+1)_{n}}{(v+2)(e)_{n}} \frac{(-x)^{n}}{n !}{ }_{3} F_{2}\left[\begin{array}{c}
-n,-v-1-n, 1-e-n ; \\
\mu,-e-n ;
\end{array}\right] .
\end{aligned}
$$

Now, if we make use of extension of Gauss summation theorem (1.8), after some simplification, we easily arrive at the desired results (3.3). This completes the proof of Theorem 3.

We also note that if one puts $\mu=1-v$, one obtains Theorem 2, and if $\mu=1+v$ one obtains Theorem 1.

\section{Special cases}

(a) In (3.1), if we set $e=v+1$, we have

$$
e^{-x} \sum_{n=0}^{\infty} \frac{x^{n}}{n !}{ }_{1} F_{1}\left[\begin{array}{c}
-n ; \\
v+1 ;
\end{array}\right]={ }_{0} F_{1}\left[\begin{array}{c}
-; \\
v+1 ;
\end{array}-x^{2}\right],
$$

which, upon using the result (1.12) reduces to the well-known result (1.15).

(b) In (3.2), if we set $e=v+1$, we have

$$
\begin{aligned}
e^{-x} & \sum_{n=0}^{\infty} \frac{(v+1)_{n}}{(1-v)_{n}} \frac{x^{n}}{n !}{ }_{1} F_{1}\left[\begin{array}{c}
-n ; \\
v+1 ;
\end{array}\right] \\
\quad= & { }_{1} F_{2}\left[\begin{array}{c}
\frac{1}{2} v+\frac{1}{2} ; \\
\frac{1}{2}, 1-\frac{1}{2} v ;
\end{array}-x^{2}\right]+\frac{2 x v}{1-v}{ }_{1} F_{2}\left[\begin{array}{c}
\frac{1}{2} v+1 ; \\
\frac{3}{2}, \frac{3}{2}-\frac{1}{2} v ;
\end{array}\right],
\end{aligned}
$$


which upon using the result (1.12) reduces to the well-known result (1.16).

(c) In (3.3), if we set $e=v+1$ so that $A=1-\mu$, we have

$$
e^{-x} \sum_{n=0}^{\infty} \frac{(1+v)_{n}}{(\mu)_{n}} \frac{(-x)^{n}}{n !}{ }_{1} F_{1}\left[\begin{array}{c}
-n ; \\
v+1 ;
\end{array}\right]={ }_{2} F_{2}\left[\begin{array}{c}
\frac{1}{2} \mu+\frac{1}{2} v, \frac{1}{2} \mu+\frac{1}{2} v+\frac{1}{2} ; \\
\mu, \mu+v ;
\end{array}\right],
$$

which upon using the result (1.12) reduces to the well-known result (1.17).

Similarly, other results can also be obtained.

\section{Competing interests}

The authors declare that they have no competing interests.

\section{Authors' contributions}

The authors have made equal contributions to each part of this article. All authors have read and approved the final manuscript.

\section{Author details}

${ }^{1}$ Department of Mathematics, School of Mathematical and Physical Sciences, Central University of Kerala, Riverside Transit Campus, Padennakkad, P.O. Nileshwar, Kasaragod, Kerala 671 328, India. ${ }^{2}$ Department of Mathematics, Institute of Mathematical Research, University Putra Malaysia (UPM), Serdang, Selangor 43400, Malaysia.

\section{Acknowledgements}

The authors are very grateful to the referees for their valuable suggestions and comments that improved the paper.

Received: 24 September 2013 Accepted: 9 January 2014 Published: 28 Jan 2014

\section{References}

1. Bailey, WN: Generalized Hypergeometric Series. Cambridge Tracts in Mathematics and Mathematical Physics, vol. 32. Cambridge University Press, Cambridge (1935). Reprinted by Stechert-Hafner, New York (1964)

2. Rainville, ED: Special Functions. Macmillan Co., New York (1960). Reprinted by Chelsea, New York (1971)

3. Slater, LJ: Generalized Hypergeometric Functions. Cambridge University Press, Cambridge (1966)

4. Kim, YS, Rakha, MA, Rathie, AK: Extensions of certain classical summation theorems for the series ${ }_{2} F_{1,3} F_{2}$, and ${ }_{4} F_{3}$ with applications in Ramanujan's summations. Int. J. Math. Math. Sci. 2010, Article ID 309503 (2010)

5. Rakha, MA, Rathie, AK: Generalizations of classical summation theorems for the series ${ }_{2} F_{1}$ and ${ }_{3} F_{2}$ with applications. Integral Transforms Spec. Funct. 22(11), 823-840 (2011)

6. Exton, H: Multiple Hypergeometric Functions. Halsted, New York (1976)

7. Prudnikov, AP, Brychkov, YA, Marichev, Ol: Integrals and Series, Volume 3: More Special Functions. Nauka, Moscow (1986) (in Russian). Translated from the Russian by GG Gould, Gordon \& Breach, New York (1990)

8. Eldelyi, A: Higher Transcendental Functions. McGraw-Hill, New York (1953)

9. Kim, YS, Rathie, AK, Paris, RB: On a new class of summation formulae involving the Laguerre polynomial. Integral Transforms Spec. Funct. 23(6), 435-444 (2012)

10. Exton, H: Summation formulae involving the Laguerre polynomials. J. Comput. Appl. Math. 100, 225-227 (1998)

11. Paris, RB: A Kummer-type transformation for a ${ }_{2} F_{2}$ hypergeometric function. J. Comput. Appl. Math. 173, 379-382 (2005)

10.1186/1687-1847-2014-43

Cite this article as: Rathie and Kılıçman: On a new class of summation formulas involving the generalized hypergeometric ${ }_{2} F_{2}$ polynomial. Advances in Difference Equations 2014, 2014:43

\section{Submit your manuscript to a SpringerOpen ${ }^{\circ}$ journal and benefit from:}

- Convenient online submission

- Rigorous peer review

- Immediate publication on acceptance

Open access: articles freely available online

- High visibility within the field

- Retaining the copyright to your article 